\title{
Ocorrência de cochonilhas em espinheira-santa (Maytenus ilicifolia Mart. ex Reissek)
}

\section{VITÓRIA, J.M.'; ZACARIAS, F.M.'; MARIOT, M.P. ${ }^{2 *}$; BARBIERI, R.L. ${ }^{3}$; PINTO, J.R. ${ }^{2}$}

${ }^{1}$ Acadêmica de Tecnologia em Gestão Ambiental, Instituto Federal Sul-rio-grandense/Campus Pelotas - Visconde da Graça, Av. Ildefonso Simões Lopes, 2791, CEP 96.060-290, Pelotas-Brasil; 'Professor do Instituto Federal Sul-rio-grandense /Campus Pelotas - Visconde da Graça, *marciomariot@gmail.com; ${ }^{3}$ Pesquisadora da Embrapa Clima Temperado, Rodovia BR 392, km 78, CEP 96.010-971, Pelotas-Brasil

\begin{abstract}
RESUMO: Maytenus ilicifolia é uma espécie medicinal nativa do Brasil que vem sendo cultivada para atender a crescente demanda do mercado. Os fatores que interferem na produção, tal como a incidência de pragas e doenças, devem ser estudados para garantir o incremento na produtividade. $O$ objetivo deste trabalho foi registrar a ocorrência e a identificação de cochonilhas associadas a 15 acessos de espinheira-santa (Maytenus ilicifolia) do Banco Ativo de Germoplasma da Embrapa Clima Temperado e do Instituto Federal Sul-rio-grandense. Foi registrada a quantidade de plantas infestadas em cada acesso e identificado, em laboratório, as espécies de cochonilhas infestantes. O número de plantas infestadas variou entre os acessos. Foram identificadas as cochonilhas Ceroplastes sp. e Saissetia oleae, família Coccidae. Dos 10 acessos infestados, $23(11,3 \%)$ plantas apresentaram infestação com Ceroplastes sp. e, apenas uma (0,5\%), com Saissetia oleae. O acesso 133 mostrou a maior incidência de Ceroplastes sp. $(33,3 \%)$, seguido do acesso $130(25,0 \%)$. O acesso 123 foi o que apresentou menor quantidade de plantas infestadas $(4,8 \%)$. Saissetia oleae ocorreu em apenas uma planta, pertencente ao acesso $136(5,6 \%)$.
\end{abstract}

Palavras-chave: Celastraceae, planta medicinal, pragas, Ceroplastes.sp, Saissetia oleae

ABSTRACT: Occurrence of mealybugs in "espinheira-santa" (Maytenus ilicifolia Mart. ex Reissek). Maytenus ilicifolia is a medicinal species native to Brazil which has been cultivated to meet the growing market demand. Factors that interfere with the production, such as the incidence of pests and diseases, should be studied to ensure increased productivity. The aim of this study was to record the occurrence and the identification of mealybugs associated with 15 "espinheira-santa" (Maytenus ilicifolia) accessions from the Active Germplasm Bank of "Embrapa Clima Temperado" and "Instituto Federal Sul-rio-grandense". The quantity of infested plants in each accession was recorded and the species of infested mealybugs were identified in the laboratory. The number of infested plants varied among accessions. The identified mealybugs were Ceroplastes sp. and Saissetia oleae, family Coccidae. Of 10 infested accessions, 23 plants $(11.3 \%)$ showed infestation with Ceroplastes sp., and only one $(0.5 \%)$ with Saissetia oleae. Accession 133 showed the highest incidence of Ceroplastes sp. (33.3\%), followed by accession $130(25.0 \%)$. Accession 123 had the least amount of infested plants (4.8\%). Saissetia oleae occurred in only one plant, belonging to accession $136(5.6 \%)$.

Key words: Celastraceae, medicinal plant, pests, Ceroplastes.sp, Saissetia oleae

\section{INTRODUÇÃO}

Maytenus ilicifolia Mart. ex Reissek (espinheira-santa), família Celastraceae, é uma espécie medicinal nativa do Brasil, com ocorrência predominantemente na Região Sul. O alto valor medicinal, com recomendação para problemas de gastrite e ulcera gástrica, tem motivado intensa ação antrópica nas populações naturais, levando a espécie a ser incluída como prioritária em programas de conservação (Mariot \& Barbieri, 2010). Parte da variabilidade genética de espinheira-santa do Rio Grande do Sul está sendo conservada, desde 2004, no Banco Ativo de Germoplasma (BAG) da Embrapa Clima Temperado e do Instituto Federal Sul-riograndense (IFSul)/Campus Pelotas - Visconde da Graça, em Pelotas, no RS.

A espinheira-santa, já cultivada comercial-

Recebido para publicação em 18/07/2011

Aceito para publicação em 02/02/2012

Rev. Bras. PI. Med., Campinas, v.15, n.2, p.176-179, 2013. 
mente no estado do Paraná, apresenta potencial para inserção na matriz agrícola também no Rio Grande do Sul, especialmente devido ao aumento da demanda após a comprovação de suas propriedades medicinais e do lançamento de medicamento à base da planta (Mariot \& Barbieri, 2006). A espécie está na lista de plantas indicadas para uso no Sistema Único de Saúde (RENISUS) (DAF/SCTIE/MS - RENISUS, 2009).

Entre as espécies nativas de interesse a serem cultivadas, Magalhães (2010) destacou a espinheira-santa. No Paraná são cultivados 66 ha da espécie, além disso, existe extrativismo nas populações naturais (Scheffer et al., 2005).

O valor de venda da espinheira-santa para os produtores varia de $R \$ 2,00$ a $R \$ 12,00$ $\mathrm{kg}^{-1}$, dependendo do nível tecnológico da produção (Cardon \& Carvalho, 2005). De acordo com os autores, a produtividade é em torno de $1.028 \mathrm{~kg}$ de folha seca ha ${ }^{-1}$ ano-1 $^{-1}$, para os produtores que utilizam tecnologia mais desenvolvida. A espinheira-santa para o consumidor é comercializada a um preço que varia entre $R \$ 20,00$ e $R \$ 290,00 \mathrm{~kg}^{-1}$.

Para inserir efetivamente a espinheira-santa na matriz agrícola, o conhecimento sobre fatores que interferem na produção, a exemplo da incidência de pragas e doenças, é fundamental para viabilizar o cultivo da espécie com incremento de produtividade.

Com relação às pragas, ainda que muitas delas não causem sérios danos às plantas de espinheira-santa, tem sido observada a ocorrência de cochonilhas, ácaros e pulgões (Magalhães, 2002; Cirio et al., 2003). Os pulgões, quando em grande infestação, causam o encarquilhamento das folhas, o ataque de formigas cortadeiras, logo após o transplante das mudas, pode prejudicar seriamente a cultura, e a infestação por mosca-branca em mudas cultivadas em viveiros causa a redução no crescimento das plantas (Montanari Jr. et al., 2004). A presença de formiga (Crematogastersp.) e gafanhoto (Xyleus sp.) em plantas de Maytenus ilicifolia, foi constatada por Leite et al. (2006), mas em baixa proporção. Cochonilhas são, entretanto, as pragas que mais têm sido relatadas em espinheira-santa. Negrelle et al. (1999), ao pesquisarem técnicas para produção de sementes de Maytenus ilicifolia, citam a presença de cochonilhas de carapaça nos ramos das plantas. Cirio et al. (2003), por sua vez, observaram a incidência de cochonilhas com carapaça e também cochonilhas cerosas em plantas cultivadas no Paraná, sendo que as cerosas foram identificadas como Ceroplastes spp.

Em uma ampla revisão sobre cochonilhas do gênero Pseudoparlatoria, família Diaspididae, Wolf (2008) registrou a ocorrência de $P$. fusca em Maytenus ilicifolia e $P$. parlatorioides em Maytenus sp. No Estado de São Paulo, Peronti et al. (2008) identificaram em Maytenus sp. a ocorrência de Ceroplastes rotundus, $C$. formicarius e $C$ communis, da sub-família Ceroplastinae Atkinson (família Coccidae). Em Viamão, no Rio Grande do Sul, Wolff et al. (2009) observaram a ocorrência de cochonilhas Coccus sp., da família Coccidae, e de Acutaspis perseae e Parlatoria pergandii, da família Diaspididae, em plantas de Maytenus ilicifolia, mas sem sintomas de dano aparente.

A fim de verificar a presença, e identificar espécies de cochonilhas ocorrentes em plantas de espinheira-santa do BAG da Embrapa Cima Temperado e do IFSul, no município de Pelotas, Rio Grande do Sul, foi desenvolvido o presente trabalho.

\section{MATERIAL E MÉTODO}

As avaliações quanto à incidência e a identificação de cochonilhas foram feitas em outubro de 2010, em 15 acessos de espinheira-santa (Maytenus ilicifolia Mart. ex Reissek) conservadas no BAG da Embrapa Clima Temperado e do IFSul, no município de Pelotas, no Rio Grande do Sul. Cada acesso compreende progênies de meios-irmãos, oriundos de uma única planta matriz. As plantas matrizes, das quais foram retiradas sementes para produzir as progênies, ocorrem em propriedades rurais dos municípios de Canguçu e Piratini, também no Rio Grande do Sul. Os acessos, a origem, e a quantidade de plantas de cada acesso, estão relacionados na Tabela 1.

TABELA 1. Acessos de espinheira-santa (Maytenus ilicifolia), origem e número de plantas do Banco Ativo de Germoplasma da Embrapa Clima Temperado e IFSul. 2010. Pelotas-RS, Brasil.

\begin{tabular}{ccc}
\hline Acesso & Munícipio de origem & Número de plantas \\
\hline 116 & Canguçu & 19 \\
117 & Canguçu & 19 \\
118 & Canguçu & 15 \\
122 & Canguçu & 21 \\
123 & Canguçu & 21 \\
127 & Piratini & 9 \\
129 & Piratini & 11 \\
130 & Piratini & 8 \\
131 & Piratini & 6 \\
132 & Piratini & 1 \\
133 & Piratini & 9 \\
134 & Piratini & 2 \\
135 & Piratini & 25 \\
136 & Piratini & 18 \\
137 & Piratini & 19 \\
\hline Total & & 203 \\
\hline
\end{tabular}


Foi verificado a ocorrência de cochonilhas em todas as plantas dos acessos do BAG. Ramos com folhas de plantas infestadas foram encaminhadas para identificação no laboratório de fitossanidade do Campus Pelotas - Visconde da Graça do IFSul. Para a identificação das espécies foi utilizada a chave proposta por Zucchi et al. (1993). As plantas que apresentavam as espécies de cochonilhas identificadas foram registradas em uma planilha e, posteriormente, foi quantificado o percentual de plantas infestadas por acesso. Cada planta foi considerada como uma repetição, e o número de repetições variou de um acesso para outro, devido à diferença quanto à disponibilidade de sementes por ocasião da coleta de germoplasma para implantação do BAG de espinheira-santa.

\section{RESULTADO E DISCUSSÃO}

Foram identificadas as espécies de cochonilhas Ceroplastes sp. e Saissetia oleae, ambas pertencentes à família Coccidae. Do total de 203 plantas avaliadas, $24(11,8 \%)$ apresentaram ocorrência de cochonilhas, sendo $23(11,3 \%)$ delas com Ceroplastes sp. e apenas uma $(0,5 \%)$ com Saissetia oleae (Tabela 2). Dos 15 acessos avaliados, apenas os acessos de número 118, 127, 131, 132 e 134 não mostraram incidência de cochonilha. A não ocorrência de cochonilha nesses acessos pode sugerir a presença de resistência genética. Ao observar plantas de espinheira-santa cultivadas no Paraná, Scheffer (2004) também

TABELA 2. Incidência de Ceroplastes sp. e Saissetia oleae em acessos de espinheira-santa (Maytenus ilicifolia) do Banco Ativo de Germoplasma da Embrapa Clima Temperado/IFSul. 2010. PelotasRS, Brasil.

\begin{tabular}{ccc}
\hline Acesso & Ceroplastes sp. (\%) & Saissetia oleae (\%) \\
\hline 116 & 15,8 & - \\
117 & 10,5 & - \\
118 & - & - \\
122 & 19,0 & - \\
123 & 4,8 & - \\
127 & - & - \\
129 & 18,2 & - \\
130 & 25,0 & - \\
131 & - & - \\
132 & - & - \\
133 & 33,3 & - \\
134 & - & - \\
135 & 8,0 & - \\
136 & 11,1 & 5,6 \\
137 & 10,5 & - \\
\hline Total & 11,3 & 0,5 \\
\hline
\end{tabular}

identificou a ocorrência de plantas resistentes a cochonilhas com ou sem carapaça e outras muito suscetíveis.

A ocorrência de cochonilhas foi considerada baixa, o que corrobora as observações de Magalhães (2002), Cirio et al. (2003) e Wolff et al. (2009) quanto ao pouco dano aparente causado pelos insetos. Entretanto, é importante o conhecimento das pragas que ocorrem em espinheira-santa, com o monitoramento da sua incidência, por ser uma espécie que vem sendo cultivada, o que, segundo Cirio et al. (2003), contribui para o aumento da probabilidade de infestações.

Os autores destacaram que a presença de cochonilhas da família Coccidae, observada em amostras de espinheira-santa, influenciaram negativamente na qualidade da matéria-prima a ser beneficiada, por propiciarem aumento da umidade, interferência nos valores de matéria orgânica estranha, alteração nos valores de cinzas totais e insolúveis em ácido, além de modificações sensoriais que inviabilizaram seu uso como droga. Estas avaliações levaram a reprovação de alguns lotes.

Considerando os 10 acessos infestados por Ceroplastes sp., o acesso 133 foi o que mostrou maior incidência dessa espécie de cochonilha $(33,3 \%)$, seguido pelo acesso $130(25,0 \%)$, sendo que o acesso 123 foi o que apresentou menor quantidade de plantas infestadas $(4,8 \%)$. Os insetos estavam presentes nos ramos das plantas.

Saissetia oleae ocorreu em apenas uma planta, pertencente ao acesso 136 (5,6\%), tanto nos ramos quanto na face abaxial das folhas, e não estava associada à outra espécie de cochonilha. Para esta planta, a infestação foi maior nas folhas do que nos ramos. Este inseto pertence ao grupo das cochonilhas com escudo e ainda não havia sido relatada a sua ocorrência em Maytenus ilicifolia, sendo este, portanto, o primeiro registro. Saissetia oleae tem a forma convexa, de coloração marrom e apresenta o corpo com carenas dorsais em forma de "H" (Zucchi et al., 1993). Outros autores relataram a ocorrência de cochonilha de escudo (carapaça) em espinheira-santa (Negrelle et al., 1999 e Cirio et al., 2003), mas sem identificação. Outras espécies de cochonilha com escudo foram identificadas por Wolff (2008) (Pseudoparlatoria fusca em Maytenus ilicifolia e Pseudoparlatoria parlatorioides em Maytenus sp) e por Wolff et al. (2009) (Coccus sp., Parlatoria pergandii e Acutaspis perseae em Maytenus ilicifolia).

Ceroplastes sp., que mostrou maior incidência nas plantas de espinheira-santa avaliadas neste trabalho, parece ser de ocorrência comum, tendo sido identificada por Cirio et al. (2003) em Maytenus ilicifolia e por Peronti et al. (2008) em 
Maytenus sp. Conhecidos popularmente por cochonilhas cerosas, Ceroplastes sp. apresentam espessa carapaça cerosa que recobre o corpo das fêmeas adultas (Peronti et al., 2008).

\section{CONCLUSÃO}

- Foram identificadas as cochonilhas Ceroplastes sp. e Saissetia oleae em Maytenus iliciolia, com baixa incidência.

- Ceroplastes sp. apresentou maior ocorrência em espinheira-santa, com maior incidência no acesso 133.

- A não ocorrência de cochonilha em alguns acessos sugere que existem genótipos resistentes.

- Saissetia oleae é relatada pela primeira vez em espinheira-santa e ocorreu apenas no acesso 136.

\section{AGRADECIMENTO}

Os autores agradecem à Fapergs e ao CNPq pela concessão de bolsa de iniciação científica às acadêmicas do curso de Tecnologia em Gestão Ambiental.

\section{REFERÊNCIA}

CARDON, L.M.; CARVALHO, R.I.N. Métodos de coleta, secagem, armazenamento, destino e condições de transporte da carqueja e da espinheira-santa na região Metropolitana de curitiba. Revista Acadêmica: ciências agrárias e ambientais, v.3, n.1, p.49-56, 2005.

CIRIO, G.M. et al. Interrelação de parâmetros agronômicos e físicos de controle de qualidade de Maytenus ilicifolia Mart. ex Reiss. (espinheira-santa) como insumo para a indústria farmacêutica. Visão acadêmica, v.4, n.2, p.67-76, 2003.

DAF/SCTIE/MS. RENISUS - Relação Nacional de Plantas Medicinais de Interesse ao SUS. DAF/SCTIEI MS - RENISUS, 2009. Disponível em: <http://portal. saude.gov.br/portal/arquivos/pdf/RENISUS.pdf>. Acesso em: 2 mar. 2012.

LEITE, G.L.D. et al. Fatores climáticos influenciam a abundância de artrópodes de plantas medicinais no Estado de Minas Gerais, Brasil. Revista Brasileira de Plantas Medicinais, v.8, n.3, p.43-51, 2006.
MAGALHÃES, P.M. Agrotecnologia para o cultivo da espinheira-santa. Plantas Medicinais, 2002. Disponível em: <http://www.cpqba.unicamp.br/plmed/ artigos/agroespsant.htm>. Acesso em: 10 jul. 2011.

MAGALHÃES, P.M. Estratégias para o mercado de plantas medicinais e aromáticas no Brasil: o exemplo da erva-baleeira. Informe Agropecuário, v.31, n.255, p.94-100, 2010.

MARIOT, M.P.; BARBIERI, R.L. Divergência genética entre acessos de espinheira-santa (Maytenus ilicifolia Mart. ex Reissek e M. aquifolium Mart.) com base em caracteres morfológicos e fisiológicos. Revista Brasileira de Plantas Medicinais, v.12, n.3, p.243-9, 2010.

MARIOT, M.P.; BARBIERI, R.L. Espinheira-santa: uma alternativa de produção para a pequena propriedade. Pelotas: Embrapa Clima Temperado (Série Documentos 177), 2006. 32p.

MONTANARI Jr. et al. Cultivo de espinheira-santa. In: REIS, M.S.; SILVA, S.R. (organizadores). Conservação e uso sustentável de plantas medicinais e aromáticas: Maytenus spp., espinheira-santa. Brasília, DF: IBAMA, 2004. p.163-80.

NEGRELLE, R.R.B. et al. Tecnologia de produção de sementes de espinheira-santa (Maytenus ilicifolia Mart. ex Reiss. - Celastraceae). Revista Brasileira de Sementes, v.21, n.1, p.76-81, 1999.

PERONTI, A.L.B.G. et al. Revisão das espécies de Ceroplastinae Atkinson (Hemiptera, Coccoidea, Coccidae) do Estado de São Paulo, Brasil. Revista Brasileira de Entomologia, v.52, n.2, p.139-181, 2008.

SCHEFFER, M.C. Espinheira-santa (Maytenus ilicifolia Mart. ex Reiss) production in the metropolitan region of Curitiba, Paraná, Brazil. In: ALEXIADES, M.N.; SHANLEY, P. (Eds.). Forest products, livelihoods and conservation: case studies of non-timber forest product systems. V.3. Bogor, Indonesia: Center for International Forestry Research, 2004, p.313-330.

SCHEFFER, M.C.; CORREAA JR, C.; Radomski, M.I. Domesticação de plantas medicinais: a experiência da "Espinheira-Santa" (Maytenus ilicifolia Mart. ex Reiss). Agrociencia, v.9, n.1 y n.2, p.53-60, 2005.

WOLFF, V.R. Revisão de Pseudoparlatoria (Hemiptera, Diaspididae). Iheringia, Série Zoologia, v.98, n.3, p.291-307, 2008.

WOLFF, et al. Cochonilhas (Hemiptera, Sternorrhyncha, Coccoidea) associadas à plantas medicinais e aromáticas. Pesquisa Agropecuária Gaúcha, v.15, n.1, p.65-8, 2009.

ZUCCHI, R.A. et al. Guia de identificação de pragas agrícolas. Piracicaba: FEALQ, 1993. 139p. 